\title{
Transient Solutions of Markov Processes by Krylov Subspaces
}

Bernard PHILIPPE and Roger B. SIDJE

\section{$\mathbf{N}^{\circ} 1989$}

Août 1993

PROGRAMME 6

Calcul scientifique, modélisation

et logiciels numériques

\section{apport \\ derecherche}





\title{
RINRIA
}

\section{Transient Solutions of Markov Processes by Krylov Subspaces*}

\author{
Bernard PHILIPPE and Roger B. SIDJE \\ Programme 6 - Calcul scientifique, modélisation et logiciel numérique \\ Projet ALADIN \\ Rapport de recherche ${ }^{\circ} 1989$ - Août 1993 - 33 pages
}

\begin{abstract}
In this note we exploit the knowledge embodied in infinitesimal generators of Markov processes to compute efficiently and economically the transient solution of continuous time Markov processes. We consider the Krylov subspace approximation method which has been analysed by Y. Saad for solving linear differential equations. We place special emphasis on error bounds and stepsize control. We discuss the computation of the exponential of the Hessenberg matrix involved in the approximation and an economic evaluation of the Padé method is presented. We illustrate the usefulness of the approach by providing some application examples.
\end{abstract}

Key-words: Markov chains, matrix exponential, Krylov subspaces, Arnoldi.

(Résumé : tsvp)

${ }^{*}$ This work was supported by an NSF-INRIA agreement. 


\section{Approximation des solutions transitoires d'un processus de Markov dans des sous-espaces de Krylov}

Résumé : Nous nous intéressons dans cet article au calcul du régime transitoire d'un processus de Markov. Un tel problème nécessite le calcul d'une exponentielle de matrice. En prenant en considération certaines propriétés inhérentes aux modèles markoviens, nous examinons l'approximation dans un espace de Krylov proposée par Y. Saad. Cela nous permet de préciser certaines caractéristiques théoriques et de définir un algorithme fiable et efficace. Nous présentons des résultats numériques attestant de la validité de la méthode.

Mots-clé : Chaînes de Markov, exponentielle d'une matrice, espaces de Krylov, méthode d'Arnoldi. 


\section{Introduction}

Problem Definition. Continuous-time Markov Chains (CTMC) are widely used in the analysis of the behavior of many physical systems. Given a system whose evolution can be modeled by a CTMC, $X=\{X(t), t \geq 0\}$ having a finite discrete state space $\Omega=$ $\{1,2, \ldots, N\}$, the transient analysis involves the computation of the state probability vector $\pi(t)=\left(\pi_{1}(t), \pi_{2}(t), \ldots, \pi_{N}(t)\right)$ where $\pi_{i}(t)=\operatorname{Prob}\{X(t)=i\}$ is the probability that the Markov process $X$ is in state $i$ at time $t$. Interesting measures (availability, reliability, performability) of the behavior of the system being modeled can then be evaluated as weighted sums of state probabilities (see, e.g, [18] and references therein). Let $Q=\left[q_{i j}\right]$ be the infinitesimal generator of $X . Q$ is also called the transition rate matrix and $q_{i j} \geq$ $0, i \neq j$, is the rate of transition from state $i$ to state $j$ and $q_{i i}=-\sum_{i \neq j} q_{i j}$.

The evolution of the state probability vector $\pi(t)$ can be mathematically represented by the Chapman-Kolmogorov system of differential equations. For convenience of notation we set

$$
A=Q^{T}, \quad v=\pi^{T}(0), \quad w(t)=\pi^{T}(t)
$$

and hence, the differential system under concern may be written as

$$
\left\{\begin{array}{llll}
\frac{d w(t)}{d t} & =A w(t), & & t \in[0, T] \\
w(0) & =v, & & \text { initial state probability vector }
\end{array}\right.
$$

and has the analytic solution

$$
w(t)=e^{t A} v
$$

Largeness, stiffness and accuracy are the three major difficulties related to the practical computation of $(2)[6,11,18]$.

Intrinsic Properties. There are a number of inherent characteristics of the model (see, e.g., [4, 21] for a general overview). We give below a list of those which are relevant for our presentation.

P1 $\mathbb{1}^{T} A=0$ where $\mathbb{1}=(1,1, \ldots, 1)^{T}$.

$\mathrm{P} 20 \leq w_{i}(t) \leq 1$ and $\|w(t)\|_{1}=\mathbb{1}^{T} w(t)=1$.

P3 0 is a simple eigenvalue of $A$ and for any non-null eigenvalue $\lambda$ of $A, \Re e(\lambda)<0$. Hence the differential system (1) is stable and has a steady state solution.

$\mathrm{P} 4$ If $\pi$ denotes the left eigenvector of $\mathrm{Q}$ associated to the eigenvalue 0 , i.e. $\pi Q=0$ then, we have $\pi^{T}=w_{\infty}=\lim _{t \rightarrow \infty} e^{t A} v$ and $A w_{\infty}=0$. The row vector $\pi$ is known as 
the stationary probability vector; i.e. $\pi_{i}$ is the probability that the Markov chain will be at state $i$ at statistical equilibrium.

Notation. Throughout this paper we will designed by $I$ an identity matrix whose size is defined according to its context. The $j$-th canonical vector is denoted by $e_{j}$ and we will also assume that its length is defined by its context. $\lambda(M)$ represents the spectrum of the matrix $M$. The integer $m$ is some prescribed constant which should not exceed $N$, the order of the principal matrix $A$. We set $\rho=\|A\|_{2}$.

The organization of the paper is as follows. In Section 2 we consider the motivations of a time-stepping integrator for the problem. Section 3 presents the basic Krylov subspace approximation method and some extensions. Section 4 is concerned with the practical computation of the exponential of the small Hessenberg matrix involved in the schemes. Section 5 discusses the preservation of probability constraints. Section 6 considers numerical issues and finally Section 7 presents some numerical examples.

\section{Integration Scheme}

One way to solve (1) consists in computing the matrix exponential $e^{t A}$. Unfortunately as pointed out in the survey paper of C. B. Moler and C. F. Van Loan [12] none of the methods proposed up to now is suitable for the general use. Moreover, for large sparse matrices, the exponential becomes full and little can be done to prevent the fill-in. Among current methods, the Taylor expansions have been at the basis of most solution techniques used in Markov chains analyzers. It is the case of the Standard Uniformization (SU) method which is probably the most widely used. Another approach referred as the Uniformized Power (UP) method which provides a new mechanism for the control of the error was recently introduced in [1] and was found to be an improvement over the SU method in time complexity for a class of stiff Markov processes. But it requires the full matrix and is not intended for large systems states. However as shown by Y. Saad [19], it is more promising to tackle the computation of the matrix exponential times a vector rather than the matrix exponential in isolation. For our concern, a consideration is that, generally system managers are interested in discrete observations

$$
w\left(t_{i}\right)_{0 \leq i \leq s+1}, \quad 0=t_{0}<t_{1}<\cdots<t_{s}<t_{s+1}=T
$$

where the observation times $\left\{t_{i}\right\}_{0 \leq i \leq s+1}$ may or may not be uniformly spaced and may or may not be known in advance. If we take $w_{i}=w\left(t_{i}\right)$ and $\tau_{i}=t_{i+1}-t_{i}$ then, we consider the integration scheme

$$
\left\{\begin{array}{l}
w_{0}=v \\
w_{i+1}=e^{\tau_{i} A} w_{i}, \quad i=0,1, \ldots, s
\end{array}\right.
$$


so that the kernel of the problem remains the operation $e^{\tau A} v$. The accuracy of the matrix exponential depends heavily on the matrix norm [22] and for an arbitrary large value of $t$, a straightforward computation of $e^{t A} v$ will certainly end up yielding unsatisfactory results. To preserve accuracy, it is necessary to use a step-by-step computation as in single-step ODE solvers.

\section{Krylov Approximation}

In this section, for ease of reading, we will drop the time variable and we will show later how to easily re-insert it in the proposed schemes.

\subsection{Arnoldi-based Schemes}

Following the observation that a truncated power series of order $m-1$, or more generally, that approximating $e^{A} v$ by $p_{m-1}(A) v$ where $p_{m-1}$ is a polynomial of degree $m-1$, will yield an element of the Krylov subspace

$$
\mathbf{K}_{m}(A, v)=\operatorname{Span}\left\{v, A v, \ldots, A^{m-1} v\right\}
$$

the approximation problem can be reformulated as finding an element of $\mathbf{K}_{m}(A, v)$ that approaches $w=e^{A} v$.

If $V_{m}=\left[v_{1}, v_{2}, \ldots, v_{m}\right]$ is a basis of the Krylov subspace $\mathbf{K}_{m}(A, v)$ then, the best approximation $\widehat{w}$ of $w$ in this subspace should satisfy

$$
\begin{aligned}
\|\widehat{w}-w\|_{2} & =\min _{x \in \mathbf{K}_{m}(A, v)}\|x-w\|_{2} \\
& =\min _{y \in \mathbb{R}^{m}}\left\|V_{m} y-e^{A} v\right\|_{2} .
\end{aligned}
$$

If we take $\beta=\|v\|_{2}$ and $v_{1}=v / \beta$ then, $v=\beta V_{m} e_{1}$ and from the above full rank least square problem the desired $\widehat{w}$ is

$$
\begin{aligned}
\widehat{w} & =V_{m}\left[\left(V_{m}^{T} V_{m}\right)^{-1} V_{m}^{T} e^{A} v\right] \\
& =V_{m}\left[\left(V_{m}^{T} V_{m}\right)^{-1} V_{m}^{T} e^{A} V_{m}\right] \beta e_{1} .
\end{aligned}
$$

If the well known Arnoldi process is used to build the basis $V_{m}$ then, at completion $V_{m}$ is orthonormal, i.e. $V_{m}^{T} V_{m}=I$, and an upper Hessenberg matrix $H_{m}$ is constructed so that

$$
\begin{aligned}
A V_{m} & =V_{m} H_{m}+h_{m+1, m} v_{m+1} e_{m}^{T} \\
V_{m}^{T} A V_{m} & =H_{m} .
\end{aligned}
$$


The optimal Krylov element in equation (6) is

$$
\widehat{w}=\beta V_{m} V_{m}^{T} e^{A} V_{m} e_{1} .
$$

Relation (9) remains unfortunately uncomfortable since it requires $e^{A}$. However we may approximate $V_{m}^{T} e^{A} V_{m}$ by $e^{V_{m}^{T} A V_{m}}$. In other words, the restriction of the exponential operator $e^{A}$ on the subspace $\mathbf{K}_{m}(A, v)$ with respect to the basis $V_{m}$ is approximated by the exponential of the restriction of the operator $A$ on the same subspace with respect to the same basis. Using (8) the computed approximation is

$$
\widehat{w} \approx \widetilde{w}=\beta V_{m} e^{H_{m}} e_{1}
$$

In relation (10) we still have to compute a matrix exponential, but the matrix $H_{m}$ involved is of modest size compared to the original matrix $A$. Additionnally, it has a special structure. The effective computation of $e^{H_{m}}$ may be perfomed using a rational approximation or any other suitable method among those presented in [12].

Therefore the approximation of $w$ by $\widetilde{w}$ involves three levels of approximations :

- considering $e^{A} v$ as an element of $\mathbf{K}_{m}(A, v)$ instead of $\mathbf{K}_{N}(A, v)$

- considering $e^{V_{m}^{T} A V_{m}}$ instead of $V_{m}^{T} e^{A} V_{m}$

- considering (rational) approximation of $e^{V_{m}^{T} A V_{m}}$.

However the technique has been successfully used. In [8] (see also references therein) it is reliably used to solve parabolic partial differential equations and in [19] the following comforting results were proved.

Theorem 3.1 (Saad 1992) Let $\beta=\|v\|_{2}, \rho=\|A\|_{2}$ and $\lambda\left(H_{m}\right)$ be the set of Ritz values of $A$ over $V_{m}$ i.e. the eigenvalues of $H_{m}$.

1. The approximation (10) is mathematically equivalent to approximating $e^{A} v$ by $p_{m-1}(A) v$ where $p_{m-1}$ is the (unique) polynomial of degree $m-1$ which interpolates the exponential function in the Hermite sense on the set $\lambda\left(H_{m}\right)$ with each Ritz value repeated according to its multiplicity and the following a priori error bound is satisfied

$$
\left\|e^{A} v-\beta V_{m} e^{H_{m}} e_{1}\right\|_{2} \leq 2 \beta \frac{\rho^{m} e^{\rho}}{m !} .
$$

2. Let

$$
\bar{H}_{m+1} \equiv\left(\begin{array}{cc}
H_{m} & 0 \\
h_{m+1, m} e_{m}^{T} & 0
\end{array}\right)
$$

The corrected approximation

$$
\widetilde{w}_{c o r}=\beta V_{m+1} e^{\bar{H}_{m+1}} e_{1}
$$

is mathematically equivalent to approximating $e^{A} v$ by $p_{m}(A) v$ where $p_{m}$ is the (unique) polynomial of degree $m$ which interpolates the exponential function in the Hermite sense 
on the set $\lambda\left(H_{m}\right) \cup\{0\}$ with each Ritz value repeated according to its multiplicity and the following a priori error bound is satisfied

$$
\left\|e^{A} v-\beta V_{m+1} e^{\bar{H}_{m+1}} e_{1}\right\|_{2} \leq 2 \beta \frac{\rho^{(m+1)} e^{\rho}}{(m+1) !} .
$$

3. Let

$$
\begin{aligned}
\phi_{0}(z) & \equiv e^{z} \\
\phi_{k}(z) & \equiv \frac{\phi_{k-1}(z)-\phi_{k-1}(0)}{z}, \quad k \geq 1, \\
& =\sum_{i=0}^{\infty} \frac{z^{i}}{(i+k) !}=\frac{1}{z^{k}}\left(e^{z}-e_{k-1}(z)\right),
\end{aligned}
$$

where $e_{k-1}(z) \equiv \sum_{i=0}^{k-1} \frac{z^{i}}{i !}$ is the $(k-1)$-st partial sum of the power series of $e^{z}$. The following expansion holds

$$
\begin{aligned}
e^{A} v & =\beta V_{m} e^{H_{m}} e_{1}+\beta h_{m+1, m} \sum_{j=1}^{k} e_{m}^{T} \phi_{j}\left(H_{m}\right) e_{1} A^{j-1} v_{m+1}+\beta A^{k} s_{m}^{k} \\
& =\beta V_{m} e^{H_{m}} e_{1}+\beta h_{m+1, m} \sum_{j=1}^{\infty} e_{m}^{T} \phi_{j}\left(H_{m}\right) e_{1} A^{j-1} v_{m+1}
\end{aligned}
$$

where

$$
s_{m}^{k} \equiv \phi_{k}(A) v_{1}-V_{m} \phi_{k}\left(H_{m}\right) e_{1} .
$$

Proof. See [19]

In correlation with the results of this theorem there are several mathematical properties. Among them we have :

- The corrected approximation (13) is simply the first truncated term in the expansion $(16)$, i.e.

$$
\widetilde{w}_{c o r}=\beta V_{m} e^{H_{m}} e_{1}+\beta h_{m+1, m} e_{m}^{T} \phi_{1}\left(H_{m}\right) e_{1} v_{m+1} .
$$

- If the minimal degree of $v$ is some integer $\nu(\nu \leq m \leq N)$ then, an invariant subspace is found and the approximation $\beta V_{\nu} e^{H_{\nu}} e_{1}$ is exact. In exact arithmetic this situation, which is usually referred as a «breakdown», happens at least when $m=N$.

- Since $V_{m}^{T}(\tau A) V_{m}=\tau H_{m}$ for any arbitrary scalar $\tau$, we have

$$
e^{\tau A} v=w(\tau) \approx \widetilde{w}(\tau)=\beta V_{m} e^{\tau H_{m}} e_{1}
$$


- The functions $\phi_{k}$ are positive, increasing and $\phi_{k+1} \leq \frac{1}{k} \phi_{k}$ in $[0, \infty)$. Moreover as $k$ increases, they become smoother. For $x \geq 0$ we have the following bounds

$$
\frac{1}{k !} \leq \phi_{k}(x) \leq \min \left(\frac{e^{x}}{k !}, \frac{e^{x}}{x^{k}}\right)
$$

In the neighborhood of the origin, the upper bound can be improved

$$
x \leq \frac{k+1}{2} \Longrightarrow \phi_{k}(x) \leq \min \left(\frac{2}{k !}, \frac{1+2 x}{k !}\right) .
$$

Indeed if $x \leq \frac{k+1}{2}$ then, the inequality $\phi_{k}(x) \leq \frac{2}{k !}$ comes directly from the definition of $\phi_{k}$. Moreover

$$
\phi_{k}(x)=\frac{1}{k !}\left(1+x \sum_{i=0}^{\infty} \frac{x^{i} k !}{(i+k+1) !}\right)
$$

But

$$
\frac{k !}{(i+k+1) !}=\frac{1}{(k+1) \cdots(i+k+1)} \leq \frac{1}{(k+1)^{i}},
$$

thus

$$
\sum_{i=0}^{\infty} \frac{x^{i} k !}{(i+k+1) !} \leq \sum_{i=0}^{\infty} \frac{1}{2^{i}}=2
$$

We can also state the following result.

Proposition 3.1 Let

$$
\begin{aligned}
\Pi_{j} & =\prod_{k=2}^{j+1} h_{k, k-1}, \forall j \geq 1 \\
D_{m} & =\operatorname{diag}\left(1, \tau \Pi_{1}, \tau^{2} \Pi_{2}, \ldots, \tau^{m-1} \Pi_{m-1}\right) \\
\widehat{H}_{m} & =D_{m}^{-1}\left(\tau H_{m}\right) D_{m} \\
& =\left(\begin{array}{ccccc}
\tau h_{11} & & & & \\
1 & \tau h_{22} & & \hat{h}_{i j} & \\
0 & 1 & \tau h_{33} & & \\
\vdots & \ddots & \ddots & \ddots & \\
0 & \ldots & 0 & 1 & \tau h_{m m}
\end{array}\right)
\end{aligned}
$$

where

$$
\hat{h}_{i j}=\tau^{j-i+1} h_{i j} \prod_{k=i+1}^{j} h_{k, k-1}
$$

Then,

$$
e^{\tau A} v=\beta V_{m} e^{\tau H_{m}} e_{1}+\beta \tau^{m} \Pi_{m} \sum_{j=1}^{\infty} e_{m}^{T} \phi_{j}\left(\widehat{H}_{m}\right) e_{1}(\tau A)^{j-1} v_{m+1}
$$


Proof. The main key for the proof is the relation

$$
e_{m}^{T} \phi_{j}\left(\tau H_{m}\right) e_{1}=\tau^{m-1} \Pi_{m-1} e_{m}^{T} \phi_{j}\left(\widehat{H}_{m}\right) e_{1}
$$

which is valid since

$$
D_{m} e_{m}=\tau^{m-1} \Pi_{m-1}, \quad D_{m}^{-1} e_{1}=1
$$

and

$$
e_{m}^{T}\left(\tau H_{m}\right)^{i} e_{1}=e_{m}^{T} D_{m} \widehat{H}_{m}^{i} D_{m}^{-1} e_{1}=\tau^{m-1} \Pi_{m-1} e_{m}^{T} \widehat{H}_{m}^{i} e_{1} .
$$

Thus using (16)

$$
\begin{aligned}
e^{\tau A} v & =\beta V_{m} e^{\tau H_{m}} e_{1}+\beta \tau^{m-1} \Pi_{m-1} \tau h_{m+1, m} \sum_{j=1}^{\infty} e_{m}^{T} \phi_{j}\left(\widehat{H}_{m}\right) e_{1}(\tau A)^{j-1} v_{m+1} \\
& =\beta V_{m} e^{\tau H_{m}} e_{1}+\beta \tau^{m} \Pi_{m} \sum_{j=1}^{\infty} e_{m}^{T} \phi_{j}\left(\widehat{H}_{m}\right) e_{1}(\tau A)^{j-1} v_{m+1}
\end{aligned}
$$

The expansion provided by this proposition takes into account the stepsize $\tau$, the order $m$ and all the subdiagonal elements of $H_{m}$. It is well known that some of these elements are very small when we tend to an invariant Krylov subspace.

\subsection{Extended Arnoldi Schemes}

\subsection{1 k-corrected Schemes}

A natural way for attempting to improve the approximation (10) is to ask whether or not more terms of expansion (15) can be accumulated. We must keep in mind that going through this expansion requires the determination of the coefficients $h_{m+1, m} e_{m}^{T} \phi_{j}\left(H_{m}\right) e_{1}$ and extra matrix-vector products. This last aspect gives rise to discussion because if we perform additional matrix-vector multiplications we may happily compute the approximated solution in the associated higher order Krylov subspace. However here we give a simple procedure to compute the desired coefficients of the expansion.

Proposition 3.2 Let $h=h_{m+1, m}$ and

$$
\bar{H}_{m+k}=\left(\begin{array}{cccccc}
H_{m} & & & & 0 & \\
h e_{m}^{T} & 0 & & & & \\
0 & 1 & 0 & & & \\
& 0 & 1 & 0 & & \\
\vdots & & \ddots & \ddots & \ddots & \\
0 & \cdots & & 0 & 1 & 0
\end{array}\right) \in \mathbb{R}^{(m+k) \times(m+k)}
$$


then

$$
e^{\bar{H}_{m+k}}=\left(\begin{array}{cccccc}
e^{H_{m}} & & & & 0 & \\
h e_{m}^{T} \phi_{1}\left(H_{m}\right) & 1 & & & & \\
h e_{m}^{T} \phi_{2}\left(H_{m}\right) & 1 / 1 ! & 1 & & & \\
& 1 / 2 ! & 1 / 1 ! & 1 & & \\
\vdots & & \ddots & \ddots & \ddots & \\
h e_{m}^{T} \phi_{k}\left(H_{m}\right) & 1 /(k-1) ! & \ldots & 1 / 2 ! & 1 / 1 ! & 1
\end{array}\right)
$$

Proof. Let us consider the block lower triangular decomposition

$$
\bar{H}_{m+k}=\left(\begin{array}{cc}
H_{m} & 0 \\
h e_{1} e_{m}^{T} & J
\end{array}\right), \quad J=\left(\begin{array}{cccc}
0 & & & 0 \\
1 & 0 & & \\
& \ddots & \ddots & \\
0 & & 1 & 0
\end{array}\right)=\left[e_{2}, \ldots, e_{k}, 0\right] .
$$

Then

$$
e^{\bar{H}_{m+k}}=\left(\begin{array}{cc}
e^{H_{m}} & 0 \\
F & e^{J}
\end{array}\right), \quad e^{J}=\left(\begin{array}{cccc}
1 & & & 0 \\
1 / 1 ! & 1 & & \\
\vdots & \ddots & \ddots & \\
1 /(k-1) ! & \ldots & 1 / 1 ! & 1
\end{array}\right), \quad F=\left(\begin{array}{c}
f_{1} \\
f_{2} \\
\vdots \\
f_{k}
\end{array}\right) .
$$

But since $\bar{H}_{m+k} e^{\bar{H}_{m+k}}=e^{\bar{H}_{m+k}} \bar{H}_{m+k}$ we have

$$
F H_{m}-J F=h\left(e_{1} e_{m}^{T} \exp \left(H_{m}\right)-\exp (J) e_{1} e_{m}^{T}\right) .
$$

Multiplying on the left by $e_{i}^{T}$ to extract the $i$-th row, we obtain the recurrence relations

$$
\left\{\begin{array}{l}
f_{1 .}=h e_{m}^{T} \phi_{1}\left(H_{m}\right), \\
f_{i .} H_{m}=f_{i-1 .}-h e_{m}^{T} \frac{1}{(i-1) !}, \quad 1<i \leq k
\end{array}\right.
$$

and therefore by induction $f_{i .}=h e_{m}^{T}\left(\phi_{i-1}\left(H_{m}\right)-\phi_{i-1}(0) I\right) H_{m}^{-1}=h e_{m}^{T} \phi_{i}\left(H_{m}\right)$.

This result shows that the desired coefficients are on the first column just below the $m$-th row of $e^{\bar{H}_{m+k}}$. These coefficients will also be used later for error estimation and stepsize control. The following result gives an a priori upper bound on the error when the $k$-corrected scheme is used.

Theorem 3.2 If the $k$-corrected scheme is used, i.e. if we approximate $w=e^{A} v$ by

$$
\widetilde{w}_{k-\text { cor }}=\beta V_{m} e^{H_{m}} e_{1}+\beta h_{m+1, m} \sum_{j=1}^{k} e_{m}^{T} \phi_{j}\left(H_{m}\right) e_{1} A^{j-1} v_{m+1}
$$

then

$$
\left\|w-\widetilde{w}_{k-\operatorname{cor}}\right\|_{2} \leq 2 \beta \frac{\rho^{(m+k)} e^{\rho}}{(m+k) !} .
$$


Moreover if we assume that $\rho \leq \frac{m+k+1}{2}$ then

$$
\left\|w-\widetilde{w}_{k-\text { cor }}\right\|_{2} \leq 4 \beta \frac{\rho^{m+k}}{(m+k) !} .
$$

Proof. The proof is an extended version of that of Theorem 4.7 in [19]. Let

$$
e_{i-1}(z) \equiv \sum_{j=0}^{i-1} \frac{z^{j}}{j !} \text { and } t_{i}(z) \equiv e^{z}-e_{i-1}(z)=\sum_{j=i}^{\infty} \frac{z^{j}}{j !} .
$$

For any polynomial $p_{m-1}$ of degree $m-1$, setting $r_{m}(z) \equiv \phi_{k}(z)-p_{m-1}(z)$, we can write

$$
s_{m}^{k} \equiv \phi_{k}(A) v_{1}-V_{m} \phi_{k}\left(H_{m}\right) e_{1}=r_{m}(A) v_{1}-V_{m} r_{m}\left(H_{m}\right) e_{1} .
$$

Thus for the polynomial defined by

$$
p_{m-1}(z) \equiv \frac{e_{m+k-1}(z)-e_{k-1}(z)}{z^{k}}=\sum_{j=0}^{m-1} \frac{z^{j}}{(j+k) !}
$$

we have

$$
\begin{aligned}
r_{m}(z) \equiv \phi_{k}(z)-p_{m-1}(z)=\frac{e^{z}-e_{k-1}(z)}{z^{k}}-\frac{e_{m+k-1}(z)-e_{k-1}(z)}{z^{k}} & =\frac{t_{m+k}(z)}{z^{k}} \\
& =\sum_{j=m}^{\infty} \frac{z^{j}}{(j+k) !} .
\end{aligned}
$$

It follows that

$$
\left\|r_{m}(A) v_{1}\right\| \leq \sum_{j=m}^{\infty} \frac{\rho^{j}}{(j+k) !}=\frac{t_{m+k}(\rho)}{\rho^{k}} .
$$

The function $\frac{t_{m+k}(\rho)}{\rho^{k}}$ is increasing and from Lemma 4.2 of [19], we know that

$$
t_{m+k}(\rho) \leq \frac{\rho^{m+k} e^{\rho}}{(m+k) !} .
$$

Using (15) and (26), since $\left\|V_{m}\right\|_{2}=1$ and $\widetilde{\rho} \equiv\left\|H_{m}\right\|_{2} \leq\|A\|_{2} \equiv \rho$ we can write

$$
\begin{aligned}
\left\|w-\widetilde{w}_{k-\text { cor }}\right\|_{2} & =\left\|\beta A^{k} s_{m}^{k}\right\|_{2} \\
& \leq \beta \rho^{k}\left(\left\|r_{m}(A) v_{1}\right\|_{2}+\left\|V_{m} r_{m}\left(H_{m}\right) e_{1}\right\|_{2}\right) \\
& \leq 2 \beta t_{m+k}(\rho) \\
& \leq 2 \beta \frac{\rho^{m+k} e^{\rho}}{(m+k) !} .
\end{aligned}
$$

If in addition we assume that $\rho \leq \frac{m+k+1}{2}$ then,

$$
t_{m+k}(\rho)=\sum_{j=0}^{\infty} \frac{\rho^{m+k+j}}{(m+k+j) !} \leq \frac{\rho^{m+k}}{(m+k) !} \sum_{j=0}^{\infty} \frac{\rho^{j}}{(m+k+1)^{j}} \leq 2 \frac{\rho^{m}}{(m+k) !} .
$$

Notice that the exponential factor $e^{\rho}$ is dropped when we analyse only the bound near the origin. This will be exploited for the selection of the first stepsize in $\S 6.3$. 


\subsubsection{Restart and Inclusion of Eigenvectors}

Another way for attempting to improve the approximation (10) is to adapt a restart. Unfortunately if restarting is a trivial matter in linear systems and eigenvalue problems, the situation here is quite different. Indeed if restarting is done, we will end up with a subspace which has nothing to do with (5). However we can obtain via Krylov subspace projection methods or any suitable method a set of real Schur vectors

$$
X=\left[x_{1}, x_{2}, \ldots, x_{\ell}\right]
$$

which spans the unique invariant subspace associated with some eigenvalues $\lambda_{1}, \lambda_{2}, \ldots, \lambda_{\ell}$ (see [9, chap. 7]). We then proceed by computing a basis which is orthogonal to $X$. Practically, this is performed by a slight modification in the Arnoldi process (see algorithm in $\S 6.4)$. Formally let us consider the decomposition

$$
v=\sum_{i=1}^{\ell} \beta_{i} x_{i}+\beta v_{1} \quad \text { where }\left\|v_{1}\right\|_{2}=1 .
$$

Using a modified Arnoldi procedure we may construct a system $\widetilde{V}_{m}$ such that $\left[X, \widetilde{V}_{m}\right]$ is a basis of $\operatorname{Span}\left\{x_{1}, x_{2}, \ldots, x_{\ell}, v_{1}, A v_{1}, \ldots, A^{m-1} v_{1}\right\}$ with $\widetilde{V}_{m}^{T} X=0$ and $\widetilde{V}_{m}^{T} \widetilde{V}_{m}=I$. We consider the approximation

$$
\widetilde{w}=\widetilde{V}_{\ell+m} e^{\widetilde{H}_{\ell+m}} \widetilde{V}_{\ell+m}^{T} v
$$

where

$$
\begin{aligned}
\tilde{V}_{\ell+m} & =\left[X, \tilde{V}_{m}\right] \\
\widetilde{H}_{\ell+m} & =\tilde{V}_{\ell+m}^{T} A \widetilde{V}_{\ell+m}=\left(\begin{array}{cc}
X^{T} A X & X^{T} A \tilde{V}_{m} \\
0 & \widetilde{V}_{m}^{T} A \widetilde{V}_{m}
\end{array}\right) \\
\tilde{V}_{\ell+m}^{T} v & =\left(\beta_{1}, \ldots, \beta_{\ell}, \beta, 0, \ldots, 0\right)^{T} .
\end{aligned}
$$

Since vectors in $X$ are real Schur vectors, $X^{T} X=I$ and $X^{T} A X$ is upper quasi-triangular i.e. triangular with possibly nonzero $2 \times 2$ blocks along the diagonal (it is primordial to choose $\ell$ such that this is achieved otherwise the scheme fails). Moreover $\widetilde{V}_{m}^{T} A \widetilde{V}_{m} \equiv \widetilde{H}_{m}$ is upper Hessenberg. In the same spirit as in Theorem 3.1 the computed approximation (27) will correspond to the Hermite interpolation of the exponential on the set $\left\{\lambda_{1}, \lambda_{2}, \ldots, \lambda_{\ell}\right\} \cup$ $\lambda\left(\widetilde{H}_{m}\right)$. We expect this extended scheme, which is a form of deflation, to be satisfactory especially when $v$ has rich components in $X$ or when the $\left\{\lambda_{i}\right\}_{i=1, \ell}$ are close to zero. We can combine it with the $k$-corrected scheme to estimate errors sharply and we point out that $X$ is computed once and is used for varying $v$ 's. Moreover the computation of $e^{\widetilde{H}_{\ell+m}}$ is done by blocks. 